Discussion Paper No. 829

\title{
DOES SELF-REGULATION OF ADVERTISEMENT LENGTH IMPROVE CONSUMER WELFARE?
}

\author{
Taisuke Matsubae \\ Noriaki Matsushima
}

January 2012

The Institute of Social and Economic Research

Osaka University

6-1 Mihogaoka, Ibaraki, Osaka 567-0047, Japan 


\title{
Does self-regulation of advertisement length improve consumer welfare?*
}

\author{
Taisuke Matsubae ${ }^{\dagger}$ \\ Competition Policy Research Center, Japan Fair Trade Commission \\ Noriaki Matsushima \\ Institute of Social and Economic Research, Osaka University
}

January 18,2012

\begin{abstract}
In Japan, TV platforms regulate themselves as to the length of the advertisements they air. Using modified Hotelling models, we investigate whether such self-regulation improves consumer and social welfare or not. When all consumers choose a single TV program (the utility functions of consumers satisfy the standard 'full-coverage' condition), self-regulation always reduces consumer welfare. It improves social welfare only if the advertisement revenue of each platform is not small and the cost parameter of investments in improving the quality of TV programs is small. When some consumers have outside options (the standard 'full-coverage' condition is not satisfied), self-regulation can benefit consumers because it increases the number of consumers who watch TV programs.
\end{abstract}

Keywords: self-regulation, program quality, advertisement volume, consumer surplus, heterogeneous consumer

JEL Classification codes: L13, L41, L82

\footnotetext{
*We are grateful to Noriyuki Doi, Hiroyuki Odagiri, Yosuke Okada, and participants in Workshop in Competition Political Research Center for discussion and comments. We especially would like to thank Koki Arai and Ayako Suzuki for their constructive discussion and comments. The views expressed herein are those of the authors and should not be interpreted as those of the Japan Fair Trade Commission.

${ }^{\dagger}$ Taisuke Matsubae, Competition Policy Research Center, Japan Fair Trade Commission, 1-1-1 Kasumigaseki, Chiyoda, Tokyo 100-8987, Japan. E-mail: fwin6544@nifty.com

†Corresponding author: Noriaki Matsushima, Institute of Social and Economic Research, Osaka University, 6-1 Mihogaoka, Ibaraki, Osaka 567-0047, Japan. Phone: (81)-6-6879-8571. Fax: (81)-6-6879-8583. E-mail: nmatsush@iser.osaka-u.ac.jp
} 


\section{Introduction}

Most nations regulate advertisements in the broadcasting industry (Anderson (2007)). There are restrictions on both the length and content of advertisements. Length restrictions are widespread in developed nations, with the exception of Japan and the US. It is not obvious whether length restrictions on advertisements are harmful to consumers. Advertisers on TV programs would like to communicate with their potential customers who are watching the programs. Those communications stimulate consumption and can benefit social welfare. TV viewers, however, would like to enjoy the program content without commercial interruptions. Those interruptions are harmful from the viewpoint of welfare. It is this trade-off that we investigate here. Especially, we take into account self-regulation of the length of advertisements in the Japanese broadcasting industry.

In the Japanese broadcasting industry, there exists a policy of self-regulation (called "Jisyu-Kisei" at Japanese) on the length of advertisements although length restrictions are not officially imposed on TV programs as they are in the US. To explain this self-regulation, we mention the basic composition of advertisements in television. In general, a television or radio advertisement is called commercial message (CM). TV programs are funded by the fees obtained from CMs. In Japan, CMs are distinguished between "spot $C M$ " and "time CM." A spot CM is an advertisement during a station break and is usually broadcasted for 15 seconds. A time $C M$ is an advertisement by a sponsor of a TV program. The National Association of Commercial Broadcasters in Japan (NAB) has imposed maximum lengths of time CMs depending on the length of the TV program. ${ }^{1}$ For example, if the length of a TV program is greater than 5 minutes and less than 10 minutes, the maximum length of all advertisements in total is 2 minutes (see Table 1). This guideline is self-regulated by the Japanese broadcasting industry.

Before we explain the model structure in this paper, we briefly describe the broadcasting industry in Japan (see Fig 1). Sponsors (private and/or public firms, etc.) offer broadcast advertisements to a platform and they pay fees for their advertisements to the platform. The platform produces the program

\footnotetext{
${ }^{1}$ The NAB is a private non-profit organization whose membership consists of 201 commercial broadcasters in Japan. It was established as a voluntary organization on July 20,1951, by 16 commercial radio companies. The NAB has been a non-profit incorporated association since April 21, 1952. Its membership now comprises all terrestrial radio and television broadcasters as well as some satellite audio and television broadcasters (this information is available on the NAB website, http://nab.or.jp/).
} 


\begin{tabular}{|c|c|}
\hline The length of a TV program & The maximum length of all advertisements in total \\
\hline 5 minutes & 1 minute \\
\hline 10 minutes & 2 minutes \\
\hline 20 minutes & 2.5 minutes \\
\hline 30 minutes & 3 minutes \\
\hline 40 minutes & 4 minutes \\
\hline 50 minutes & 5 minutes \\
\hline 60 minutes & 6 minutes \\
\hline
\end{tabular}

Table 1: The criteria imposed by NAB for advertisement length

with the advertisement fees. ${ }^{2}$ The platforms supply TV programs to consumers. The advertising fee often depends on the time the program airs, the length of this advertisement, the expected number of viewers of the program, and so on. Platforms often take the following position on self-regulation: "In the absence this self-regulation, consumer welfare will diminish because the length of advertisements per program would rapidly increase. This self-regulation can be a beneficial commitment not to increase the length of advertisements." We consider whether or not this claim is plausible.

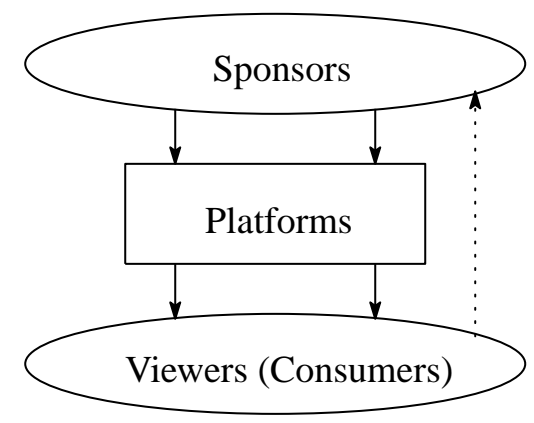

Figure 1: The image of this market.

We present a theoretical model to discuss how self-regulation in a free-to-air broadcasting industry affects the quality of TV programs, consumers, and social welfare. Basically, we follow the model setting in Gabszewicz et al. (2001, 2004). We incorporate self-regulation and heterogeneity of consumer pref-

\footnotetext{
${ }^{2}$ In Japan, intermediaries (advertisement agencies including "Dentsu" and "Hakuhodo") usually coordinate the transactions between sponsors and platforms.
} 
erences for advertisements into the model used by Gabszewicz et al. (2001, 2004). The model setting is as follows. Consumers are distributed on a line segment as in Hotelling (1929). Two platforms are respectively located at one of the edges in the line segment. The location of each consumer represents his/her preference for the platforms. Each platform determines the quality of its program and the length of advertisements. Those two factors respectively generate additional gains and losses for consumers. Each platform incurs costs to improve its TV program. An increase in the length of advertisements on a platform decreases the number of viewers. Some consumers take into account only the quality of TV programs or only the length of advertisements in each platform. That is, there are three types of consumers; those who take into account (i) quality and advertisement, (ii) only quality, and (iii) only advertisement. The revenue of each platform is proportionally correlated to the total number of viewers times the length of the advertisements (Gabszewicz et al. (2001, 2004)). The maximum advertisement length in each platform is taken to be the maximum allowed under self-regulation. The platforms set the level of self-regulation so as to maximize their joint profits.

We show that self-regulation always reduces consumer welfare. ${ }^{3}$ We also show that it improves social welfare only if per-advertisement revenue is not small and the cost parameter of quality investment for TV programs is small. Under these necessary conditions, each platform excessively engages in quality investment if they did not impose self-regulation. Self-regulation works as a credible commitment not to invest more. This limits the quality investment costs, although the benefits to consumers decrease. The former positive effect can dominate the latter negative one. We also show that the advertisement regulation imposed by a benevolent regulator can decrease consumer welfare even though it improves social welfare.

The study of advertisement in broadcasting industries has been extensive in the past decade. ${ }^{4}$ Most of these contributions focus on the combination of advertisement and horizontal product differentiation among private platforms in two-sided markets: Gabszewicz et al. (2004) study relationship between program substitutability and the degree of advertisement aversion; Anderson and Coate (2005) consider whether or not the advertisement level is socially optimal; Gantman and Shy (2007) focus on the effects of

\footnotetext{
${ }^{3}$ An extended setting shows a slightly different result.

${ }^{4}$ See the comprehensive surveys by Anderson (2007) and Anderson and Gabszewitcz (2006) about advertisement in the media.
} 
the improvement in advertisement quality. Peitz and Valletti (2008) compare the levels of advertisement intensities and program differentiation under pay-tv and free-to-air; Crampes et al. (2009) consider the effects of advertisement on entry in the media industry. González-Maestre and Martínez-Sánchez (2010) consider the role of a publicly owned platform and program quality in the free-to-air broadcasting industry. González-Maestre and Martínez-Sánchez (2011) consider the role of the endogenous choice of quality programs in a mixed duopoly market.

In the context of adverting regulation, Anderson (2007) consider the regulation of television advertisement by public authority. Stühmeier and Wenzel (2010) study the effects of symmetric and asymmetric advertisement regulation (advertisement cap) on competition for viewers and advertisers in a duopoly framework where a public and a private broadcaster compete. While those papers consider a regulated advertisement level that is a maximizer of social welfare, we focus on the self-regulated advertisement level, in which this level is a maximizer of joint profits among platforms.

The rest of the paper is organized as follows: Section 2 presents a model and the main result. Section 3 extends the basic model; we allow consumers to not watch TV programs. Section 4 concludes the paper.

\section{The model with various types of consumers}

We assume that two platforms are each located at one extreme of the linear market of length 1 . In this linear market, there exist tree types of consumers (see Fig. 2). Each consumer can choose either platform 1 or 2 .

We define each type of consumer as follows.

Type 1: There are consumers indexed by $x \in[0,1]$ and distributed uniformly along the linear market. The mass of consumers is 1 . The utility of consumer $x$ if she or he watches platform $i \in\{1,2\}$ is given as

$$
u\left(q_{i}, a_{i}, x\right)= \begin{cases}\mu+q_{1}-k a_{1}-t x & \text { if } i=1, \\ \mu+q_{2}-k a_{2}-t(1-x) & \text { if } i=2,\end{cases}
$$

where $\mu$ is a positive constant and sufficiently large (this assumption ensures that each consumer chooses one of the platforms), $q_{i}$ is the content quality of platform $i, a_{i}$ is the volume of advertisement, and $t$ represents the degree of substitutability between the two platforms. We assume that $t$ is a constant positive 
real number and that $k$ is the parameter representing the disutility per unit of advertisement and is a constant positive real number.

Type 2: There are consumers indexed by $y \in[0,1]$ and distributed uniformly along the linear market. The mass of consumers is $\lambda_{a}$ We assume that $\lambda_{a}$ is a positive real number. The utility of consumer $y$ if (s)he watches platform $i \in\{1,2\}$ is given as

$$
u\left(a_{i}, y\right)= \begin{cases}\mu-k a_{1}-t y & \text { if } i=1 \\ \mu-k a_{2}-t(1-y) & \text { if } i=2\end{cases}
$$

Type 3: There are consumers indexed by $z \in[0,1]$ and distributed uniformly along the linear market. The mass of consumers is $\lambda_{q}$. We assume that $\lambda_{q}$ is a positive real number. The utility of consumer $z$ if (s)he watches platform $i \in\{1,2\}$ is given as

$$
u\left(q_{i}, z\right)= \begin{cases}\mu+q_{1}-t z & \text { if } i=1 \\ \mu+q_{2}-t(1-z) & \text { if } i=2 .\end{cases}
$$

Consumers in Type 1 get (dis)utility from both the quality of the program and the volume of advertisement, consumers in Type 2 get (dis)utility only from the volume of advertisement, and consumers in Type 3 get utility only from the quality of the program.

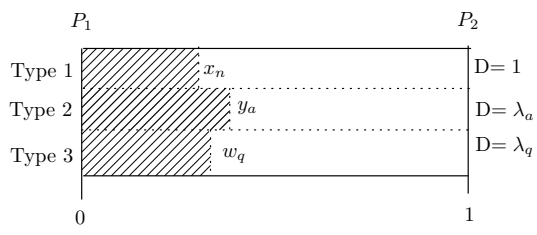

Figure 2: Image of this model

We begin to calculate each location of indifferent consumers with respect to each type. The location of indifferent type 1 consumers, $x_{n}$, is given as,

$$
x_{n}\left(a_{1}, a_{2}, q_{1}, q_{2}\right)=\frac{t+q_{1}-q_{2}-k a_{1}+k a_{2}}{2 t} .
$$

The location of indifferent type 2 consumers, $y_{a}$, is given as,

$$
y_{a}\left(a_{1}, a_{2}\right)=\frac{t-k a_{1}+k a_{2}}{2 t} .
$$


The location of indifferent type 3 consumers, $z_{q}$, is given as,

$$
z_{q}\left(q_{1}, q_{2}\right)=\frac{t+q_{1}-q_{2}}{2 t}
$$

The gross profit of each platform consists of the advertisement revenue. We assume that one unit of view for advertisement generates the revenue $\gamma$, a positive constant. This assumption means that the advertisement market is perfectly competitive (see Gabszewicz et al. $(2001,2004)$ ). The total units of view for advertisement shown in platform $i$ is the volume of advertisement times the number of audiences:

$$
\begin{aligned}
& Q_{1}\left(a_{1}, a_{2}, q_{1}, q_{2}\right)=a_{1}\left(x_{n}\left(a_{1}, a_{2}, q_{1}, q_{2}\right)+\lambda_{a} y_{a}\left(a_{1}, a_{2}\right)+\lambda_{q} w_{q}\left(q_{1}, q_{2}\right)\right) \\
& Q_{2}\left(a_{1}, a_{2}, q_{1}, q_{2}\right)=a_{2}\left(1-x_{n}\left(a_{1}, a_{2}, q_{1}, q_{2}\right)+\lambda_{a}\left(1-y_{a}\left(a_{1}, a_{2}\right)\right)+\lambda_{q}\left(1-w_{q}\left(q_{1}, q_{2}\right)\right)\right) .
\end{aligned}
$$

Each platform can improve the quality of its program, $q_{i}$, by investments. We assume that the investment cost for platform $i$ is $\phi q_{i}^{2}$, where $\phi$ is a positive real number. The profits of platforms 1 and 2 are given by

$$
\begin{aligned}
& \pi_{1}\left(a_{1}, a_{2}, q_{1}, q_{2}\right)=\gamma Q_{1}\left(a_{1}, a_{2}, q_{1}, q_{2}\right)-\phi q_{1}^{2}, \\
& \pi_{2}\left(a_{1}, a_{2}, q_{1}, q_{2}\right)=\gamma Q_{2}\left(a_{1}, a_{2}, q_{1}, q_{2}\right)-\phi q_{2}^{2} .
\end{aligned}
$$

We consider two cases: (i) the platforms do not self-regulate on their advertisement volumes; (ii) the platforms impose self-regulation on their advertisement volumes. In the first case, the game runs as follows. First, each platform simultaneously sets the quality of its program, $q_{i}$. Second, given the values of $q_{i}(i=1,2)$, they simultaneously set the volumes of advertising, $a_{i}(i=1,2)$. In the second case, the game runs as follows. First, the platforms determine the upper bound of $a_{i}, a_{r}$, to maximize their joint profits. Second, each platform simultaneously sets the quality of its program, $q_{i}$. Third, given the values of $q_{i}(i=1,2)$ and $a_{r}$, they simultaneously set the volumes of advertising, $a_{i} \in\left[0, a_{r}\right](i=1,2)$. We solve the games by backward induction.

\subsection{The case of no self-regulation in advertising}

We consider a case in which each platform can freely choose the volume of advertising. 
In the second stage, given the qualities of their programs, the reaction function of platform $i$ derived by its first-order condition and its optimal volume of advertising are given as

$$
\begin{aligned}
& a_{i}\left(a_{j}\right)=\frac{t\left(1+\lambda_{a}+\lambda_{q}\right)+\left(1+\lambda_{q}\right)\left(q_{i}-q_{j}\right)+k\left(1+\lambda_{a}\right) a_{j}}{2 k\left(1+\lambda_{a}\right)}, \\
\rightarrow & a_{i}\left(q_{i}, q_{j}\right)=\frac{3 t\left(1+\lambda_{a}+\lambda_{q}\right)+\left(1+\lambda_{q}\right)\left(q_{i}-q_{j}\right)}{3 k\left(1+\lambda_{a}\right)}, i, j=1,2, i \neq j .
\end{aligned}
$$

Substituting $a_{i}(i=1,2)$ into the profit functions, we solve the optimal qualities of their programs. We assume that

$$
\phi>\gamma\left(1+\lambda_{q}\right)^{2} /\left(18 k t\left(1+\lambda_{a}\right)\right) \equiv \underline{\phi}^{\mathrm{non}} .
$$

Note that under this condition, the second-order conditions are satisfied with respect to the profit functions.

The reaction function of platform $i$ derived by its first-order condition and the optimal quality of platform $i$ 's program, $q_{i}^{*}$, are,

$$
\begin{aligned}
& q_{i}\left(q_{j}\right)=\frac{3 t \gamma\left(1+\lambda_{q}\right)\left(1+\lambda_{a}+\lambda_{q}\right)-\gamma\left(1+\lambda_{q}\right)^{2} q_{j}}{18 k t\left(1+\lambda_{a}\right) \phi-\gamma\left(1+\lambda_{q}\right)^{2}}, \\
\rightarrow & q_{i}^{*}=\frac{\gamma\left(1+\lambda_{q}\right)\left(1+\lambda_{a}+\lambda_{q}\right)}{6 k\left(1+\lambda_{a}\right) \phi} .
\end{aligned}
$$

Substituting $q_{i}^{*}(i=1,2)$ into $a_{i}\left(q_{i}, q_{j}\right)$, we have the optimal advertising volume of platform $i$ :

$$
a_{i}^{*}=\frac{t\left(1+\lambda_{a}+\lambda_{q}\right)}{k\left(1+\lambda_{a}\right)} .
$$

We study the relationship between $q_{i}^{*}$ and $\lambda_{a}$. We illustrate how the optimal quality of program and the optimal volume of advertising change when the number of consumers of type $2, \lambda_{a}$, changes.

\section{Proposition 2.1.}

(i) An increase in $\lambda_{a}$ (an increase in the mass of Type 2 consumers) lowers program quality.

(ii) An increase in $\lambda_{a}$ (an increase in the mass of Type 2 consumers) lowers the volume of advertising.

The intuition behind this proposition is as follows. Type 2 consumers only care about the volume of advertising. An increase in the mass of Type 2 consumers means that consumers become more elastic 
with regard to their dislike for advertising. To meet changes consumer taste, each platform decreases its volume of advertising. As a result, the per-consumer revenue of each platform from advertising decreases and then the incentive of each platform to engage in quality investment diminishes.

We study the relationship between $q_{i}^{*}$ and $\lambda_{q}$. We illustrate how the optimal quality of a program and the optimal volume of advertising change when the number of consumers of Type $3, \lambda_{q}$, changes.

\section{Proposition 2.2.}

(i) An increase in $\lambda_{q}$ (an increase in the mass of Type 3 consumers) improves program quality.

(ii) An increase in $\lambda_{q}$ (an increase in the mass of Type 3 consumers) increases the volume of advertising.

The intuition behind this proposition is as follows. Type 3 consumers only care about program quality. In other words, those consumers do not take into account the volume of advertising. An increase in the mass of Type 3 consumers means that consumers become less elastic in relation to changes in advertising volume. The increase in $\lambda_{q}$ allows the platforms to increase their advertising volumes. As a result, the per-consumer revenue of each platform from advertising increases and then the incentive of each platform to improve program quality improves.

We study consumer and social welfare under $q^{*}$ and $a^{*}$. The entire consumer surplus $(C S)$ is the total consumer surplus for each type. Each consumer surplus of Type 1, Type 2, and Type 3 is denoted as $C S_{n}$, $C S_{a}$, and, $C S_{q}$, respectively. Each consumer surplus is calculated as:

$$
\begin{aligned}
C S_{n}^{*} & =\mu+\int_{0}^{x_{n}^{*}}\left(q_{1}^{*}-k a_{1}^{*}-t z\right) d z+\int_{x_{n}^{*}}^{1}\left(q_{2}^{*}-k a_{2}^{*}-t(1-z)\right) d z, \\
C S_{a}^{*} & =\lambda_{a}\left(\mu-\int_{0}^{y_{a}^{*}}\left(k a_{1}^{*}+t z\right) d z-\int_{y_{a}^{*}}^{1}\left(k a_{2}^{*}+t(1-z)\right) d z\right), \\
C S_{q}^{*} & =\lambda_{q}\left(\mu+\int_{0}^{w_{q}^{*}}\left(q_{1}^{*}-t z\right) d z+\int_{w_{q}^{*}}^{1}\left(q_{2}^{*}-t(1-z)\right) d z\right) .
\end{aligned}
$$

Note that $C S=(2.6)+(2.7)+(2.8)$. Hence, $C S$ is given as

$$
C S^{*}=\mu\left(1+\lambda_{a}+\lambda_{q}\right)+\frac{\left(1+\lambda_{a}+\lambda_{q}\right)\left(2 \gamma\left(1+\lambda_{q}\right)^{2}-15 k t\left(1+\lambda_{a}\right) \phi\right)}{12 k\left(1+\lambda_{a}\right) \phi} .
$$

We call $C S^{*}$ the consumer surplus without self-regulation of advertising. 
The total platforms' profits $\left(\pi^{*}=\pi_{1}^{*}+\pi_{2}^{*}\right)$ are given as,

$$
\pi^{*}=\frac{\gamma\left(1+\lambda_{a}+\lambda_{q}\right)^{2}\left(18 k t\left(1+\lambda_{a}\right) \phi-\lambda\left(1+\lambda_{q}\right)^{2}\right)}{18 k^{2}\left(1+\lambda_{a}\right)^{2} \phi} .
$$

We call $\pi^{*}$ the total profit without self-regulation for advertising.

We now calculate social welfare, $W$, which is defined as the sum of both platforms' profits and the total consumer surplus, $\pi^{*}+C S^{*}$ :

$$
W^{*}=\pi^{*}+C S^{*}
$$

We call $W^{*}$ social welfare without self-regulation for advertisement.

\subsection{The case of self-regulation for advertisement}

We consider the case in which the volumes of advertisement are regulated by the programmers. We assume that the volumes of advertisement must be lower than or equal to $a_{r}$. We first solve the case by assuming that $a_{r}$ is binding for the platforms in the third stage. After we derive the full game, we confirm the condition in which $a_{r}$ is really binding in equilibrium.

We suppose that $a_{r}$ is binding for the platforms in the third stage. Anticipating the third stage outcome, we determine their respective qualities. The first-order condition of platform $i$ with respect to $q_{i}$ and the outcome are given as:

$$
\frac{\gamma\left(1+\lambda_{q}\right) a_{r}-4 t \phi q_{i}}{2 t}=0 \rightarrow q_{i}=\frac{a_{r} \gamma\left(1+\lambda_{q}\right)}{4 t \phi}
$$

Given the quality of programs, we derive the optimal self-regulated advertisement level, $a_{r}^{*}$. The joint profits are given as

$$
\pi_{1}+\pi_{2}=\gamma a^{r}\left(1+\lambda_{a}+\lambda_{q}\right)-2 \phi\left(\frac{a_{r} \gamma\left(1+\lambda_{q}\right)}{4 t \phi}\right)^{2}
$$

$a_{r}^{*}$ is given as

$$
a_{r}^{*}=\frac{4 t^{2}\left(1+\lambda_{a}+\lambda_{q}\right) \phi}{\gamma\left(1+\lambda_{q}\right)^{2}} .
$$


We consider the condition such that the $a_{r}^{*}$ is binding for the platforms. This condition means that the advertising level without self-regulation is higher than that with self-regulation. Thus, we consider the region of $\phi$ such that $a^{*}-a_{r}^{*}>0$. The region is given as

$$
\phi<\frac{\gamma\left(1+\lambda_{q}\right)^{2}}{4 k t\left(1+\lambda_{a}\right)} \equiv \bar{\phi} .
$$

In this region, the optimal quality of the program under self-regulation for platform $i, q_{i r}^{*}$ is given as,

$$
q_{i r}^{*}=\frac{t\left(1+\lambda_{a}+\lambda_{q}\right)}{1+\lambda_{q}} .
$$

We state how the optimal qualities of programs and the volumes of advertisement change when each type of consumers increases. We omit the proof because the proof can be shown by using the previous method.

\section{Proposition 2.3.}

(i) An increase in $\lambda_{a}$ (an increase in the mass of Type 2 consumers) increases program quality.

(ii) An increase in $\lambda_{a}$ (an increase in the mass of Type 2 consumers) increases the volumes of advertising.

(iii) An increase in $\lambda_{q}$ (an increase in the mass of Type 3 consumers) decreases program quality.

(iv) An increase in $\lambda_{q}$ (an increase in the mass of Type 3 consumers) decreases the volumes of advertising.

The properties of this proposition are quite different from those of the previous two propositions. We summarize the two propositions in Table 2. We explain the mechanism behind this proposition.

They cooperatively determine the upper bound of $a_{i}$ at $a_{r}$. In other words, they do not compete in the advertisement levels. This implies that the demand of Type 2 consumers for platform $i$ is fixed at $\lambda_{a} / 2(i=1,2)$ under all quality and advertisement levels. An increase in $\lambda_{a}$ just enhances the demand of consumers for both platforms. Because the demand of Type 2 consumers is fixed by their self-regulation, they have an incentive to raise the regulation level $a_{r}$ (per consumer advertisement revenue). The increase in $a_{r}$ enhances the incentives of both platforms to improve the qualities of their programs. 


\begin{tabular}{|c|rl|rl|}
\hline & \multicolumn{2}{|c|}{ Increase in type 2 consumers } & \multicolumn{2}{|c|}{ Increase in type 3 consumers } \\
\hline$a_{i}^{*}$ & $\downarrow$ & (Prop.2.1.(ii)) & $\uparrow$ & (Prop.2.2.(ii)) \\
\hline$a_{r}^{*}$ & $\uparrow$ & (Prop.2.3.(ii)) & $\downarrow$ & (Prop.2.3.(iv)) \\
\hline$q_{i}^{*}$ & $\downarrow$ & (Prop.2.1.(i)) & $\uparrow$ & (Prop.2.2.(i)) \\
\hline$q_{i r}^{*}$ & $\uparrow$ & (Prop.2.3.(i)) & $\downarrow$ & (Prop.2.3.(iii)) \\
\hline
\end{tabular}

Table 2: Summary of Propositions 2.1., 2.2., and 2.3.

This mechanism does not work in cases (iii) and (iv). Type 3 consumers do not take into account the upper bound of the advertisement level, $a_{r}$. In other words, the self-regulation does not affect the choices of those consumers. An increase in $\lambda_{q}$ just enhances the demand of consuemrs for both platforms. To meet this demand expansion, they would have a high incentive to enhance the qualities of their programs if they did not impose the upper bound of the advertisement level. They anticipate that the demand expansion does not expand the total demand for their programs. From the viewpoint of their joint profits, the aggressive efforts to improve their program qualities have only a cannibalization effect on their profits. To mitigate their aggressive incentives, the platforms lower the upper bound of the advertisement level, $a_{r}$. The decrease in $a_{r}$ diminishes the incentives of both platforms to improve the qualities of their programs.

Under $\phi<\gamma\left(1+\lambda_{q}\right)^{2} /\left(4 k t\left(1+\lambda_{a}\right)\right)$, platform $i$ 's profit is given as,

$$
\pi_{i r}^{*}=\frac{t^{2}\left(1+\lambda_{a}+\lambda_{q}\right)^{2} \phi}{\left(1+\lambda_{q}\right)^{2}}
$$

Profitability of self-regulation We check the condition under which self-regulation is profitable for the platforms, $\pi_{i r}^{*}-\pi_{i}^{*}>0$. A simple calculation leads to the following inequality:

$$
\phi<\frac{(3-\sqrt{5}) \gamma\left(1+\lambda_{q}\right)^{2}}{12 k t\left(1+\lambda_{a}\right)} \equiv \bar{\phi}^{\mathrm{non}} .
$$

Note that, $\bar{\phi}^{\text {non }}<\bar{\phi}$. That is, a binding constraint $a<a_{r}$ does not always improve the profits of the platforms. We assume that the following condition is satisfied with respect to $\phi$ throughout this section. ${ }^{5}$

$$
\underline{\phi}^{\text {non }}<\phi<\bar{\phi}^{\text {non }}
$$

\footnotetext{
${ }^{5} \phi^{\text {non }}$ means that this region of $\phi$ differs from region of $\phi$ in section 3 .
} 
We calculate the consumer surplus with self-regulation. Substituting $a_{r}^{*}$ and $q_{i r}^{*}$ for (2.6), (2.7), and (2.8), and summing to those, we have

$$
C S_{r}^{*}=\left(1+\lambda_{a}+\lambda_{q}\right) \mu+\frac{t\left(1+\lambda_{a}+\lambda_{q}\right)\left(3 \gamma\left(1+\lambda_{q}\right)^{2}-16 k t\left(1+\lambda_{a}\right) \phi\right)}{4 \gamma\left(1+\lambda_{q}\right)^{2}} .
$$

The total profit of the platforms with self-regulation is given as,

$$
\pi_{r}^{*}=\frac{2 t^{2}\left(1+\lambda_{a}+\lambda_{q}\right)^{2} \phi}{\left(1+\lambda_{q}\right)^{2}}
$$

As a result, social welfare with self-regulation is $W_{r}^{*}=C S_{r}^{*}+\pi_{r}^{*}$.

$$
W_{r}^{*}=\left(1+\lambda_{a}+\lambda_{q}\right) \mu+\frac{t\left(1+\lambda_{a}+\lambda_{q}\right)\left(3 \gamma\left(1+\lambda_{q}\right)^{2}-8 t\left(2 k\left(1+\lambda_{a}\right)-\gamma\left(1+\lambda_{a}+\lambda_{q}\right)\right) \phi\right)}{4 \gamma\left(1+\lambda_{q}\right)^{2}} .
$$

\subsection{Self-regulation vs. no self-regulation}

We compare between the consumer surplus with self-regulation and that without self-regulation. Let $\triangle C S$ be $C S^{*}-C S_{r}^{*}$. A simple calculation leads to the following proposition:

Proposition 2.4. $\triangle C S$ is a positive. Thus, the consumer surplus without self-regulation is higher than that with self-regulation.

Self-regulation reduces the costs incurred by watching advertisements. This is beneficial. Because self-regulation in itself does not expand the total demand for programs, the benefit is not so strong. On the other hand, it reduces the incentives of the platforms to engage in quality investments $\left(q_{i r}^{*}<q_{i}^{*}\right)$. The latter negative effect dominates the former positive one.

We compare the difference between the social surplus without self-regulation and that with selfregulation. Note that the profit of each platform under self-regulation is higher than that under no selfregulation in $\phi \in\left(\underline{\phi}^{\text {non }}, \bar{\phi}^{\text {non }}\right)$. Let $\triangle W$ be $W^{*}-W_{r}^{*}$. We characterize this difference by $\gamma$ and $\phi$.

\section{Proposition 2.5.}

(i) If $0<\gamma<11 k\left(1+\lambda_{a}\right) /\left(1+\lambda_{a}+\lambda_{q}\right)$, $\triangle W$ is positive.

(ii) If $11 k\left(1+\lambda_{a}\right) /\left(1+\lambda_{a}+\lambda_{q}\right)<\gamma$; 
(a) If $\underline{\phi}^{\text {non }}<\phi<\underline{\phi}^{*}$, where $\underline{\phi}^{*}$ is the minimum solution such that $\triangle W=0, \triangle W$ is negative;

(b) If $\underline{\phi}^{*}<\phi<\bar{\phi}^{\text {non }}, \triangle W$ is positive.

The self-regulation improved social welfare compared with non-self-regulation when $\gamma / k$ was not small and $\phi$ was small enough. This is because each platform excessively engages in its investment if $\phi$ is small enough. Self-regulation controls their overinvestment problem. This is beneficial from the viewpoint of social welfare. Note that the overinvestment problem is not so serious when $\gamma$ is small enough. A small $\gamma$ means that per-viewer revenue is small. This implies that the returns from quality investments are small. That is, under a small $\gamma$, the platforms do not have strong incentives to engage in quality investments.

\subsection{Comparison of social welfare: self-regulation and welfare maximization}

We consider the volume of advertising that maximizes social welfare. In this case, the platforms must choose the advertising levels determined by a benevolent government. To solve the optimal volume of advertising, we calculate the equilibrium quality levels of their programs given the regulated advertising levels (defined it by $a_{g}$ ). The optimal quality of each program $q_{i}^{* *}(a)$ is given by

$$
q_{i}^{* *}\left(a_{g}\right)=\frac{a_{g} \gamma\left(1+\lambda_{a}\right)}{4 t \phi} .
$$

We substitute the optimal quality into $\pi_{1}, \pi_{2}$, and $C S$. Let the social welfare function be denoted as $W\left(a_{g}\right)=\pi_{1}+\pi_{2}+C S$, where

$$
\begin{aligned}
W\left(a_{g}\right)= & \pi_{1}+\pi_{2}+C S \\
= & \frac{a_{g} \gamma\left(8 t^{2} \phi\left(1+\lambda_{a}+\lambda_{q}\right)-\gamma\left(1+\lambda_{q}\right)^{2} a_{g}\right)}{8 t^{2} \phi} \\
& \quad+\left(1+\lambda_{a}+\lambda_{q}\right) \mu+\left(\frac{-t^{2}\left(1+\lambda_{a}+\lambda_{q}\right) \phi+a_{g}\left(\gamma\left(1+\lambda_{q}\right)^{2}-4 k t\left(1+\lambda_{a}\right) \phi\right)}{4 t \phi}\right) .
\end{aligned}
$$

We evaluate $a_{g}^{* *} \in \arg \max W\left(a_{g}\right)$. Let $a_{g}^{* *}$ be the optimal volume of advertising. Differentiating $W\left(a_{g}\right)$ with respect to $a_{g}$, and solving the derivative with respect to $a$, we have:

$$
a_{g}^{* *}=\frac{t\left(-4 k t\left(1+\lambda_{a}\right) \phi+\gamma\left(\left(1+\lambda_{q}\right)^{2}+4 t\left(1+\lambda_{a}+\lambda_{q}\right) \phi\right)\right)}{\gamma^{2}\left(1+\lambda_{q}\right)^{2}} .
$$

From the result, we have the following proposition: 


\section{Proposition 2.6.}

(i) If $\gamma<\left(k\left(1+\lambda_{a}\right)\right) /\left(1+\lambda_{a}+\lambda_{q}\right)$, a $a_{g}^{* *}$ is higher than $a_{r}^{*}$.

(ii) If $\gamma>\left(k\left(1+\lambda_{a}\right)\right)\left(1+\lambda_{a}+\lambda_{q}\right)$, $a_{g}^{* *}$ is lower than $a_{r}^{*}$.

When $\gamma$ is small, per-unit revenue is small. The incentives of quality investments are weak. The benevolent government has an incentive to encourage the platforms to invest more. A higher value of $a_{g}$ is a way to increase per-unit revenue from advertisement.

We consider $C S$ at $a_{g}^{* *}$. Substituting $a_{g}^{* *}$ for (2.6), (2.7), and (2.8) and summing up the results, we can derive the consumer surplus (denoted by $C S^{* *}$ ). We compare $C S_{r}^{*}$ with $C S^{* *}$, leading to the following proposition:

Proposition 2.7. $C S^{* *}$ is higher than $C S_{r}^{*}$.

The benevolent government takes into account not only the total profit but also the consumer surplus, although the platforms take into account only their profits when they self-regulate. The former objective fixes the distortion of self-regulation.

We compare the difference between the profits of the platforms in the cases of self-regulation and welfare-maximizing regulation.

Proposition 2.8. $\pi^{* *}$ is lower than $\pi_{r}^{*}$.

This result is just the flip-side of the previous proposition. From the propositions, the self-regulation of advertisement by the platforms improves only their own profits.

\subsection{The comparison between social welfare in the absence of self-regulation and that in welfare-maximizing regulation}

The consumer surplus is lower under conditions of self-regulation than under those with welfare-maximizing regulation. This begs the question. Is welfare-maximizing regulation is better for consumers than no regulation? We compare the difference between the consumer surplus under welfare-maximization regulation and that without regulation. The result leads to the following proposition: 


\section{Proposition 2.9.}

(i) If $5\left(1+\lambda_{a}\right) k /\left(2\left(1+\lambda_{a}+\lambda_{q}\right)\right)<\gamma, C S^{*}>C S^{* *}$.

(ii) If $49\left(1+\lambda_{a}\right) k /\left(22\left(1+\lambda_{a}+\lambda_{q}\right)\right)<\gamma \leq 5\left(1+\lambda_{a}\right) k /\left(2\left(1+\lambda_{a}+\lambda_{q}\right)\right)$,

(a) if $\underline{\phi}^{\text {non }}<\phi<\phi^{\star}, C S^{*}>C S^{* *}$;

(b) if $\phi^{\star}<\phi<\bar{\phi}^{\text {non }}, C S^{*}<C S^{* *}$, where $\phi^{\star}$ is $\phi$ such that $C S^{*}=C S^{* *}$.

(iii) If $\gamma \leq 49\left(1+\lambda_{a}\right) k /\left(22\left(1+\lambda_{a}+\lambda_{q}\right)\right), C S^{*}<C S^{* *}$.

This is related to the explanation for Proposition 2.6. When $\gamma$ is small, per-unit revenue is small. The incentives to improve quality are weak. A benevolent government has an incentive to encourage platforms for investments. A higher value of $a_{g}$ is a way to increase per-unit revenue from advertising. This improves the consumer surplus.

\section{Consumers have outside options}

We consider the case in which some consumers have outside options. There are two types of consumers who can choose not to watch TV programs. In this market, there are three types of consumers (see Figs. 3 and 4).

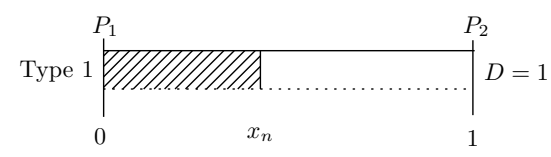

Figure 3: Demand of Type 1

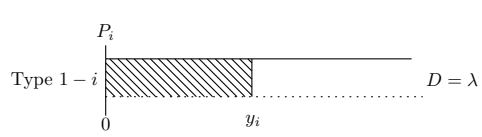

Figure 4: Demand of Type $1-i$

Type 1 consumers are the same as those in the previous section. We introduce two types of consumers that were not considered in the previous sections. We call those types 'Type 1-i' $(i=1,2)$. Type 1-i is formally defined as follows.

Type 1-i: There are consumers indexed by $y_{i} \in[0, \infty)$ and distributed uniformly along the 'half-line' as depicted in Figure 4. The mass of consumers is $\lambda$. This is a positive real number. The utility of consumer 
$y_{i}$ if she or he watches platform $i \in\{1,2\}$ is given as

$$
u\left(q_{i}, a_{i}, y_{i}\right)=v+q_{i}-k a_{i}-t y_{i}
$$

where $v$ is the reservation utility for watching a program but is not large. If her or his utility is negative, she or he does not watch program $i$. We consider the location of indifferent consumers, $y_{i}$, such that either watching platform $i$ or not watching platform $i$ is indifferent:

$$
y_{i}=\frac{v+q_{i}-k a_{i}}{t} .
$$

$x_{n}$ is the location of indifferent Type 1 consumers such that watching either platform 1 or platform 2 is indifferent. Hence, the total units of view for advertising shown in platform $i$ is the volume of advertising times the number of consumers:

$$
\begin{aligned}
& Q_{1}\left(a_{1}, a_{2}, q_{1}, q_{2}\right)=a_{1}\left(x_{n}\left(a_{1}, a_{2}, q_{1}, q_{2}\right)+\lambda y_{1}\left(a_{1}, q_{1}\right)\right), \\
& Q_{2}\left(a_{1}, a_{2}, q_{1}, q_{2}\right)=a_{2}\left(1-x_{n}\left(a_{1}, a_{2}, q_{1}, q_{2}\right)+\lambda y_{2}\left(a_{2}, q_{2}\right)\right) .
\end{aligned}
$$

Therefore, the profits of platform $i$ are given by

$$
\pi_{i}=\gamma Q_{i}\left(a_{1}, a_{2}, q_{1}, q_{2}\right)
$$

\subsection{The case without self-regulation for the volume of advertising}

We consider a case where each platform can freely choose a volume of advertising.

We begin to calculate the equilibrium qualities of programs and the volumes of advertising in this market. Given the qualities of their programs, the first-order condition of platform $i$ is

$$
a_{i}=\frac{2 \lambda v+t+(1+2 \lambda) q_{i}-q_{j}+k a_{j}}{2 k(1+\lambda)} .
$$

Each volume of advertisement is as follows:

$$
a_{i}\left(q_{i}, q_{j}\right)=\frac{(3+4 \lambda)(t+2 v \lambda)+\left(1+8 \lambda+8 \lambda^{2}\right) q_{i}-(1+2 \lambda) q_{j}}{k\left(3+16 \lambda+16 \lambda^{2}\right)} .
$$


We now consider the situation in which each platform can improve the quality of its programs. We assume that the investment cost of platform $i$ is $\phi q_{i}^{2}$, where $\phi$ is a positive real number. The profit of platform $i$ is as follows:

$$
\pi_{i}\left(q_{1}, q_{2}\right)=\gamma Q_{i}\left(a_{i}\left(q_{1}, q_{2}\right), a_{j}\left(q_{1}, q_{2}\right), q_{1}, q_{2}\right)-\phi q_{i}^{2}, i, j=1,2, j \neq i
$$

We assume that the second-order conditions of the platforms are satisfied:

$$
\frac{\gamma(1+2 \lambda)\left(1+8 \lambda+8 \lambda^{2}\right)^{2}}{2 k t(1+4 \lambda)^{2}(3+4 \lambda)^{2}}<\phi .
$$

The first-order condition of platform $i$ is

$$
q_{i}\left(q_{j}\right)=\frac{\gamma(1+2 \lambda)\left(1+8 \lambda+8 \lambda^{2}\right)\left((3+4 \lambda)(t+2 \lambda v)-(1+2 \lambda) q_{j}\right)}{2 k t(1+4 \lambda)^{2}(3+4 \lambda)^{2} \phi-\gamma(1+2 \lambda)\left(1+8 \lambda+8 \lambda^{2}\right)^{2}} .
$$

The optimal quality of platform $i, q_{i}^{*}$, is

$$
q_{i}^{*}=\frac{\gamma(1+2 \lambda)(t+2 v \lambda)\left(1+8 \lambda+8 \lambda^{2}\right)}{2\left(k t(1+4 \lambda)^{2}(3+4 \lambda) \phi-\gamma \lambda(1+2 \lambda)\left(1+8 \lambda+8 \lambda^{2}\right)\right)} .
$$

Hence, the optimal advertisement volume of platform $i, a_{i}^{*}$, is given as

$$
a_{i}^{*}=\frac{t(1+4 \lambda)(3+4 \lambda)(t+2 v \lambda) \phi}{k t(1+4 \lambda)^{2}(3+4 \lambda) \phi-\gamma \lambda(1+2 \lambda)\left(1+8 \lambda+8 \lambda^{2}\right)} .
$$

Note that we use the same symbol as in the previous section for the optimal program quality and the volume of advertising.

Under $a_{i}^{*}$ and $q_{i}^{*}, y_{i}$ is given as

$$
y_{i}^{*}=\frac{2 k(1+4 \lambda)(3+4 \lambda)((1+2 \lambda) v-t) \phi+\gamma(1+2 \lambda)\left(1+8 \lambda+8 \lambda^{2}\right)}{2\left(k t(1+4 \lambda)^{2}(3+4 \lambda) \phi-\gamma \lambda(1+2 \lambda)\left(1+8 \lambda+8 \lambda^{2}\right)\right)} .
$$

Note that there exist Type 1-i consumers who watch platform $i\left(y_{i}>0\right)$ if $v>t /(1+2 \lambda)$.

We study the welfare properties under $q^{*}$ and $a^{*}$. The consumer surplus of Type 1 (resp. 1-i) is denoted as $C S_{n}$ (resp. $C S_{o}$ ). Each consumer surplus is calculated as

$$
\begin{aligned}
& C S_{n}^{*}=\frac{\gamma(1+2 \lambda)(2 t+t \lambda+4 v \lambda)\left(1+8 \lambda+8 \lambda^{2}\right)-k t(1+4 \lambda)(3+4 \lambda)(8 \lambda v+(5+4 \lambda) t) \phi}{4\left(k t(1+4 \lambda)^{2}(3+4 \lambda) \phi-\gamma \lambda(1+2 \lambda)\left(1+8 \lambda+8 \lambda^{2}\right)\right)}, \\
& C S_{o}^{*}=\frac{t\left(\gamma(1+2 \lambda)\left(1+8 \lambda+8 \lambda^{2}\right)+2 k((1+2 \lambda) v-t)\left(3+16 \lambda+16 \lambda^{2}\right) \phi\right)^{2}}{4\left(k t(1+4 \lambda)^{2}(3+4 \lambda) \phi-\gamma \lambda(1+2 \lambda)\left(1+8 \lambda+8 \lambda^{2}\right)\right)^{2}} .
\end{aligned}
$$


$C S^{*}=C S_{n}^{*}+C S_{o}^{*}$ (see (3.2) and (3.3)). The total platforms' profits $\left(\pi^{*}=\pi_{1}^{*}+\pi_{2}^{*}\right)$ are given as,

$$
\pi^{*}=\frac{\gamma(1+2 \lambda)(t+2 v \lambda)^{2} \phi\left(2 k t\left(3+16 \lambda+16 \lambda^{2}\right)^{2} \phi-\gamma(1+2 \lambda)\left(1+8 \lambda+8 \lambda^{2}\right)^{2}\right)}{2\left(k t(1+4 \lambda)^{2}(3+4 \lambda) \phi-\gamma \lambda(1+2 \lambda)\left(1+8 \lambda+8 \lambda^{2}\right)\right)^{2}} .
$$

We now calculate social welfare, $W$, defined as the sum of profits and consumer surplus, $W^{*}=C S^{*}+\pi^{*}$.

\subsection{The case of self-regulation of advertising volume}

We consider the case in which the volumes of advertising are self-regulated. Hence, we assume that the volumes of advertising are restricted to common $a_{r}$ for both platforms. Each platform chooses only the quality level of its program given $a_{r}$. The first-order condition of $\pi_{i}$ with respect to $q_{i}$ is given as

$$
\frac{\partial \pi_{i}}{\partial q_{i}}=\frac{a_{r} \gamma+2 a_{r} \gamma \lambda-4 q_{i} t \phi}{2 t}=0 .
$$

Solving (3.4) with respect to $q_{i}$, we obtain, for $i=1,2$,

$$
q_{i}=\frac{a_{r} \gamma(1+2 \lambda)}{4 t \phi} .
$$

Given the qualities of their programs, we solve the self-regulated advertising level, $a_{r}^{*}$ :

$$
a_{r}^{*}=\frac{4 t(t+2 v \lambda) \phi}{16 k t \lambda \phi+\left(1-4 \lambda^{2}\right) \gamma} .
$$

We only consider the case in which self-regulation is binding, that is, $a_{r}^{*}<a^{*}$. We have the conditions as follows.

$$
\phi<\frac{\gamma(1+2 \lambda)^{2}(3+8 \lambda)}{4 k t(1+4 \lambda)(3+4 \lambda)} .
$$

We call the region of $\phi$ the 'binding condition'. Under this condition, the optimal qualities of the programs under self-regulation, $q_{r}^{*}$, are:

$$
q_{r}^{*}=\frac{\gamma(1+2 \lambda)(t+2 v \lambda)}{16 k t \lambda \phi+\left(1-4 \lambda^{2}\right) \gamma} .
$$

We restrict the region of $\phi$ such that $\phi$ satisfies both the second-order conditions and the binding condition in this section. Thus,

$$
\underline{\phi}:=\frac{\gamma(1+2 \lambda)\left(1+8 \lambda+8 \lambda^{2}\right)^{2}}{\left(2 k t(1+4 \lambda)^{2}(3+4 \lambda)^{2}\right)}<\phi<\frac{\gamma(1+2 \lambda)^{2}(3+8 \lambda)}{4 k t(1+4 \lambda)(3+4 \lambda)}=: \bar{\phi} .
$$


We calculate each consumer surplus with self-regulation. The consumer surplus of Type 1 (resp. 1-i) is denoted as $C S_{r} n$ (resp. $C S_{r o}$ ) in the case of self-regulation.

$$
\begin{aligned}
& C S_{r n}^{*}=\mu-\frac{16 k t(t+4 t \lambda+2 v \lambda) \phi-\gamma(1+2 \lambda)(3 t+2 t \lambda+8 v \lambda)}{4\left(16 k t \lambda \phi+\gamma\left(1-4 \lambda^{2}\right)\right)}, \\
& C S_{r o}^{*}=\frac{(4 k t(2 \lambda v-t) \phi+\gamma(t+v)(1+2 \lambda))^{2}}{t\left(16 k t \lambda \phi+\gamma\left(1-4 \lambda^{2}\right)\right)^{2}}
\end{aligned}
$$

We denote the consumer surplus with self-regulation as $C S_{r}^{*}:=C S_{r n}^{*}+C S_{r o}^{*}$.

\subsection{Comparison of welfare: self-regulation and non-self-regulation}

We compare the consumer surplus of self-regulation with that without self-regulation. Let $\triangle C S$ be the difference between $C S^{*}$ and $C S_{r}^{*}, \triangle C S \equiv C S^{*}-C S_{r}^{*}$. We characterize the relationship between consumer welfare in the two cases by $\phi$.

\section{Proposition 3.1.}

(i) If $\underline{\phi}<\phi<\underline{\phi}^{*}, \triangle C S$ is positive, where $\underline{\phi}^{*}$ is the minimum value that satisfies $C S^{*}=C S_{r}^{*}$. Thus, the consumer surplus in the case of non-self-regulation is higher than that in the case of self-regulation.

(ii) If $\underline{\phi}^{*}<\phi<\bar{\phi}, \triangle C S$ is negative.

Proposition 3.1 is different from proposition 2.7. If there exist consumers with outside options, the consumer surplus may be improved by self-regulation of advertising. In the previous section, selfregulation in itself does not expand the total demand for the programs. In this setting, self-regulation expands the total demand for the programs. This is also a benefit to consumers. Because the platforms aggressively engage in quality investment when $\phi$ is small, self-regulation significantly reduces the investment levels when $\phi$ is small. That is, when $\phi$ is small, the negative effect dominates the positive one. Note that, self-regulation does not always reduce the incentives of the platforms to engage in quality investments. 


\section{Concluding remarks}

In many countries, there are restrictions on both the length and content of advertisements. In the Japanese broadcasting industry, there are self-regulation guidelines (called "Jisyu-Kisei" in Japannease) on the length of advertisements, although length restrictions are not officially imposed on TV programs as they are in the US. We theoretically investigate how the Japanese broadcasting industry's self-regulation of the advertising length affects consumer and social welfare.

The results and the policy implications are as follows. When all consumers choose a single TV program (the utility functions of consumers satisfy the standard 'full-coverage' condition), then selfregulation always reduces consumer welfare. It improves social welfare only if the advertisement revenue of each TV platform is not small and the cost parameter of improving the quality of TV programs is small. When some consumers have outside options (the standard 'full-coverage' condition is not satisfied), the self-regulation can benefit consumers because it increase the number of consumers who watch TV programs. The results mean that self-regulation does not work as a useful tool to improve consumer welfare but does work as a collusive device to maintain the profits of TV platforms. This implies that we need to inspect broadcasters' cooperative conduct even when it seems to benefit consumers.

Although the current model does not include competition for gathering advertisers, we can extend it to that with this kind of competition. That is, we can investigate a broadcasting industry with two-sided competition ( Rochet and Tirole (2003) and Armstrong and Wright (2007)). Although we expect that this extension would complicate the analysis, this would give us interesting insights. This is a considerable future research.

\section{References}

Anderson, S. P. (2007) "Regulation of Television Advertisement" in The Economic Regulation of Broadcasting Markets Evolving Technology and Challenges for Policy, edited by Paul Seabright and Jorgen von Hagen, Cambridge University Press. 
Anderson, S. P. and S. Coate (2005) "Market Provision of Broadcasting: A Welfare Analysis" Review of Economic Studies 72(4), 947-972.

Anderson, S. P. and J. J. Gabszewicz (2006) "The Media and Advertisement: A Tale of Two-sided Markets, in Victor A. Ginsburgh and David Throsby, Editors," in Handbook of the Economics of Art and Culture, Volume 1, Elsevier B.V.

Armstrong, M. and J. Wright (2007) "Two-sided Markets, Competitive Bottlenecks and Exclusive Contracts" Economic Theory 32(2), 353-380.

Crampes, C., C. Haritchabalet and B. Jullien (2009) "Advertisement, Competition and Entry in Media Industrie" Journal of Industrial Economics 57(1), 7-31.

Gabszewicz, J. J., D. Laussel, and N. Sonnac (2001) "Press Advertisement and the Ascent of the 'Pensée Unique" European Economic Review 45(4-6), 641-651.

Gabszewicz, J. J., D. Laussel, and N. Sonnac (2004) "Programming and Advertisement Competition in the Broadcasting Industry" Journal of Economics and Management Strategy 13(4), 657-669.

Gantman, N. and O. Shy (2007) "Broadcasting Competition and Advertisement Quality: Two-sided Market Approach" mimeo.

González-Maestre, M. and F. Martínez-Sánchez (2010) “The Role of Program Quality and Publicly-owned Platforms in the Free to Air Broadcasting Industry” Working Paper. Serie AD 2010-19.

González-Maestre, M. and F. Martínez-Sánchez (2011) "Platform Quality and Advertisement Regulation in Broadcasting Markets" mimeo.

Hotelling, H. (1929) “Stability in Competition” Economic Journal 39(153), 41-57.

Peitz, M. and T. M. Valletti (2008) "Content and Advertisement in the Media: Pay-tv versus Free-to-air" International Journal of Industrial Organization 26(4), 949-965. 
Rochet, J.-C., J. Tirole (2003) "Platform Competition in Two-Sided Markets" Journal of the European Economic Association 1(4), 990-1029.

Stühmeier, T. and T. Wenzel (2010) "Regulating Advertisement in the Presence of Public Service Broadcasting" mimeo. 


\section{Technical Appendix}

\section{A Proofs in Section 2}

\section{Proof of Proposition 2.1}

We begin to prove (1). We partially differentiate the optimal quality of a program with respect to $\lambda_{a}$ and get

$$
\frac{\partial q_{i}^{*}}{\partial \lambda_{a}}=-\frac{\gamma \lambda_{q}\left(1+\lambda_{q}\right)}{6 k\left(1+\lambda_{a}\right)^{2} \phi}<0 .
$$

We prove (2). We partially differentiate the optimal volume for advertising with respect to $\lambda_{a}$ and get

$$
\frac{\partial a_{i}^{*}}{\partial \lambda_{a}}=-\frac{t \lambda_{q}}{k\left(1+\lambda_{a}\right)^{2}}<0
$$

\section{Proof of Proposition 2.2}

We begin to prove (1). We partially differentiate the optimal quality of a program with respect to $\lambda_{q}$ and get

$$
\frac{\partial q_{i}^{*}}{\partial \lambda_{q}}=\frac{2 \gamma+\gamma \lambda_{a}+2 \gamma \lambda_{q}}{6 k \phi+6 k \lambda_{a} \phi}>0 .
$$

We prove (2). We partially differentiate the optimal volume for advertising with respect to $\lambda_{q}$ and get

$$
\frac{\partial a_{i}^{*}}{\partial \lambda_{q}}=\frac{t}{k\left(1+\lambda_{a}\right)}>0 .
$$

\section{Proof of Proposition 2.4}

Calculating $\triangle C S$, we get

$$
\frac{\left(1+\lambda_{a}+\lambda_{q}\right)\left(\gamma^{2}\left(1+\lambda_{q}\right)^{4}-12 k t \gamma\left(1+\lambda_{a}\right)\left(1+\lambda_{q}\right)^{2} \phi+24 k^{2} t^{2}\left(1+\lambda_{a}\right)^{2} \phi^{2}\right)}{6 k \gamma\left(1+\lambda_{a}\right)\left(1+\lambda_{q}\right)^{2} \phi} .
$$

If $\gamma^{2}\left(1+\lambda_{q}\right)^{4}-12 k t \gamma\left(1+\lambda_{a}\right)\left(1+\lambda_{q}\right)^{2} \phi+24 k^{2} t^{2}\left(1+\lambda_{a}\right)^{2} \phi^{2}>0$, then $\triangle C S>0$.

We focus on this numerator. Note that this numerator is a quadratic function of $\phi$. This function is convex because the second derivative with respect to $\phi$ is positive. The discriminant is positive. Solving this equation with respect to $\phi$, we get the lower root $\phi^{*}$ as follows:

$$
\underline{\phi}^{*}:=\frac{12 k t \gamma\left(1+\lambda_{a}\right)\left(1+\lambda_{q}\right)^{2}-\sqrt{3} k t \gamma\left(1+\lambda_{a}\right)\left(1+\lambda_{q}\right)^{2}}{48 k^{2} t^{2}\left(1+\lambda_{a}\right)^{2}} .
$$


Note that, we have imposed the upper bound of $\phi$ at $\bar{\phi}^{\text {non }}=(3-\sqrt{5}) \gamma\left(1+\lambda_{q}\right)^{2} / 12 k t\left(1+\lambda_{a}\right)$. We estimate the difference between $\bar{\phi}^{\text {non }}$ and $\underline{\phi}^{*}$. The difference is given as

$$
\bar{\phi}^{n o n}-\underline{\phi}^{*}=\frac{(\sqrt{3}-\sqrt{5}) \gamma\left(1+\lambda_{q}\right)^{2}}{12 k t\left(1+\lambda_{a}\right)}<0 .
$$

As a result, in the imposed region of $\phi$, the numerator is a positive. We simply illustrate the idea of the proof (Fig. 5).

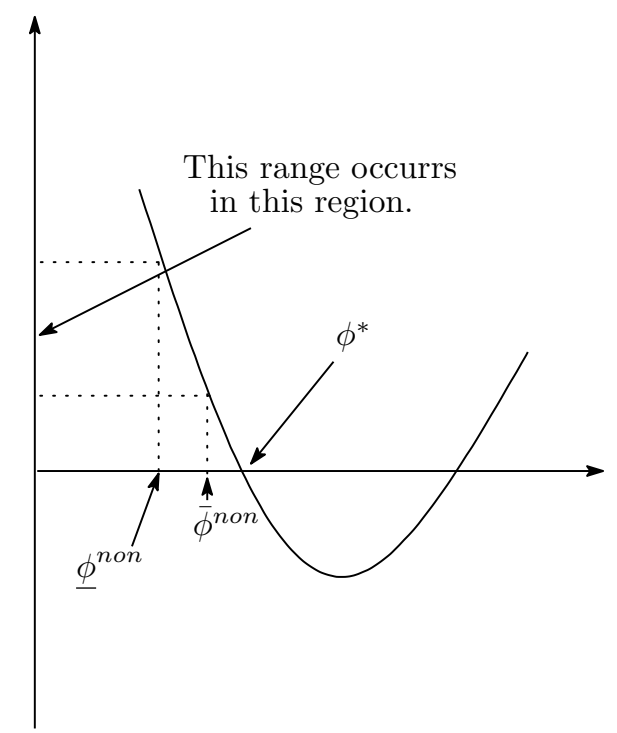

Figure 5: The image of this proof

\section{Proof of Proposition 2.5}

$\triangle W$ is given as

$$
\begin{aligned}
& \frac{1}{18 k^{2} \gamma\left(1+\lambda_{a}\right)^{2}\left(1+\lambda_{q}\right)^{2} \phi} \times \\
&\left(1+\lambda_{a}+\lambda_{q}\right)\left(\gamma^{2}\left(1+\lambda_{q}\right)^{4}\left(3 k\left(1+\lambda_{a}\right)-\gamma\left(1+\lambda_{a}+\lambda_{q}\right)\right)-18 k t \gamma\left(1+\lambda_{a}\right)\left(1+\lambda_{q}\right)^{2}\left(2 k\left(1+\lambda_{a}\right)\right.\right. \\
&\left.\left.\quad-\gamma\left(1+\lambda_{a}+\lambda_{q}\right)\right) \phi+36 k^{2} t^{2}\left(1+\lambda_{a}\right)^{2}\left(2 k\left(1+\lambda_{a}\right)-\gamma\left(1+\lambda_{a}+\lambda_{q}\right)\right) \phi^{2}\right) .
\end{aligned}
$$


We focus on the numerator of this fraction. If the numerator is positive, then $\triangle W$ is also positive. Thus, social welfare with self-regulation is lower than that without self-regulation. Let $A(\phi)$ be this numerator

$$
\begin{array}{r}
A(\phi):=\left(1+\lambda_{a}+\lambda_{q}\right)\left(\gamma^{2}\left(1+\lambda_{q}\right)^{4}\left(3 k\left(1+\lambda_{a}\right)-\gamma\left(1+\lambda_{a}+\lambda_{q}\right)\right)-18 k t \gamma\left(1+\lambda_{a}\right)\left(1+\lambda_{q}\right)^{2}\left(2 k\left(1+\lambda_{a}\right)\right.\right. \\
\left.\left.-\gamma\left(1+\lambda_{a}+\lambda_{q}\right)\right) \phi+36 k^{2} t^{2}\left(1+\lambda_{a}\right)^{2}\left(2 k\left(1+\lambda_{a}\right)-\gamma\left(1+\lambda_{a}+\lambda_{q}\right)\right) \phi^{2}\right)
\end{array}
$$

Note that $A(\phi)$ is a quadratic function with respect to $\phi$. The coefficient of $\phi^{2}$ is positive if and only if $2 k\left(1+\lambda_{a}\right) /\left(1+\lambda_{a}+\lambda_{q}\right)>\gamma$. The extreme value of $A(\phi)$ is $\phi^{\prime}=\gamma\left(1+\lambda_{q}\right)^{2} / 4 k t\left(1+\lambda_{a}\right) . \phi^{\prime}$ is larger than $\bar{\phi}^{\text {non }}$. Therefore, we have the following lemma:

Lemma A.1. 1. If $2 k\left(1+\lambda_{a}\right) /\left(1+\lambda_{a}+\lambda_{q}\right)>\gamma$, then $A(\phi)$ is minimized when $\phi^{\prime}=\gamma\left(1+\lambda_{q}\right)^{2} / 4 k t(1+$ $\left.\lambda_{a}\right)$. Thus, this function is monotonically decreasing in $\phi \in\left[\underline{\phi}^{\text {non }}, \bar{\phi}^{\text {non }}\right]$.

2. If $2 k\left(1+\lambda_{a}\right) /\left(1+\lambda_{a}+\lambda_{q}\right)<\gamma$, then $A(\phi)$ is maximized when $\phi^{\prime}=\gamma\left(1+\lambda_{q}\right)^{2} / 4 k t\left(1+\lambda_{a}\right)$. Thus, this function is monotonically increasing in $\phi \in\left[\underline{\phi}^{\text {non }}, \bar{\phi}^{\text {non }}\right]$.

We find that $A(\phi)$ is monotonically decreasing or increasing in the imposed region of $\phi$.

We prove in turn the case of (1) in proposition 2.5. We begin to consider the case in which $A(\phi)$ is a convex function. Thus, $2 k\left(1+\lambda_{a}\right) /\left(1+\lambda_{a}+\lambda_{q}\right)>\gamma$. Substituting $\bar{\phi}^{\text {non }}$ for $A(\phi)$, we get

$$
\left(1+\lambda_{a}+\lambda_{q}\right) k \gamma^{2}\left(1+\lambda_{a}\right)\left(1+\lambda_{q}\right)^{4}
$$

Clearly, the equation is a positive. Note that $\phi^{\prime}$ is larger than $\bar{\phi}^{\text {non }}$. By the lemma A.1 if $2 k(1+$ $\left.\lambda_{a}\right) /\left(1+\lambda_{a}+\lambda_{q}\right)>\gamma$, then $\triangle W$ is positive.

Next, we consider the case in which $A(\phi)$ is a concave function. Hence, substituting $\underline{\phi}^{\text {non }}$ for $A(\phi)$, we have

$$
-\frac{1}{9} \gamma^{2}\left(1+\lambda_{q}\right)^{4}\left(-11 k+\gamma-11 k \lambda_{a}+\gamma \lambda_{a}+\gamma \lambda_{q}\right)
$$

If $\gamma<11 k\left(1+\lambda_{a}\right) /\left(1+\lambda_{a}+\lambda_{q}\right)$, then the above equation is positive. Therefore, if $2 k\left(1+\lambda_{a}\right) /(1+$ $\left.\lambda_{a}+\lambda_{q}\right)<\gamma<11 k\left(1+\lambda_{a}\right) /\left(1+\lambda_{a}+\lambda_{q}\right)$, then $\triangle W$ is positive. From those facts, if $11 k\left(1+\lambda_{a}\right) /(1+$ $\left.\lambda_{a}+\lambda_{q}\right)>\gamma$, then $\triangle W$ is positive. Therefore, in the region of $\gamma$, social welfare without self-regulation is higher than that with self-regulation. 
We prove the case of (2) in Proposition 2.5. If $11 k\left(1+\lambda_{a}\right) /\left(1+\lambda_{a}+\lambda_{q}\right)<\gamma$, then $\triangle W$ is negative at $\underline{\phi}^{\text {non }}$. On the other hand, when $11 k\left(1+\lambda_{a}\right) /\left(1+\lambda_{a}+\lambda_{q}\right)<\gamma, \triangle W$ is positive at $\bar{\phi}^{\text {non }}$ and monotonically increasing in $\phi$ by lemma A.1. Thus, at $\phi$ such that $\triangle W=0$, the sign of $\triangle W$ changes from negative to positive. Thus, in $\underline{\phi}^{\text {non }}<\phi<\underline{\phi}^{*}$, social welfare with self-regulation is higher than that without selfregulation. Otherwise, social welfare with self-regulation is lower than that without self-regulation. We illustrate the sketch of proof with simple figures (Figs. 6, 7, and 8).
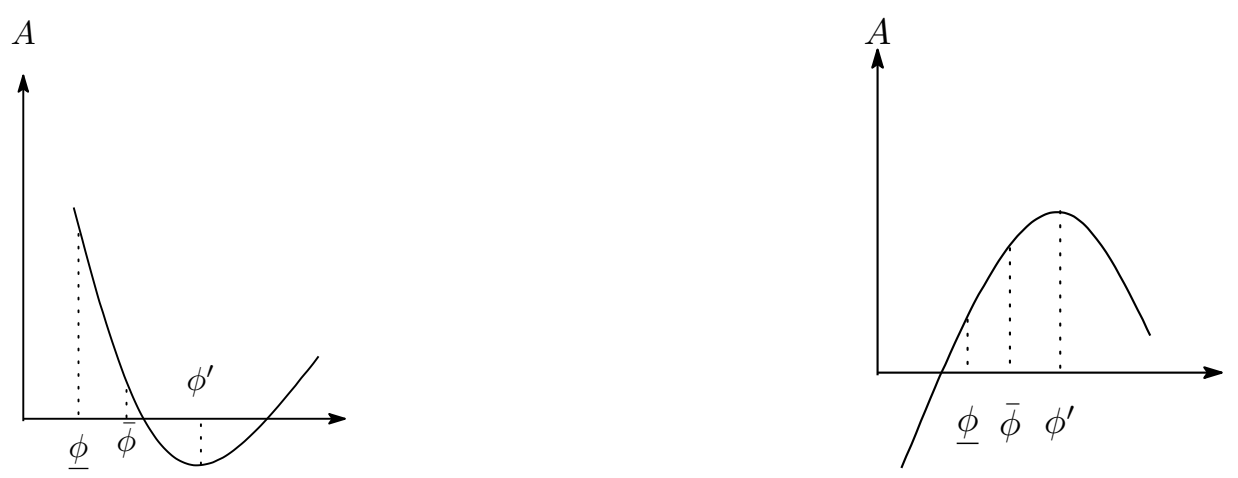

Figure 6: $2 k\left(1+\lambda_{a}\right) /\left(1+\lambda_{a}+\lambda_{q}\right)>\gamma:$ sketch of (1)

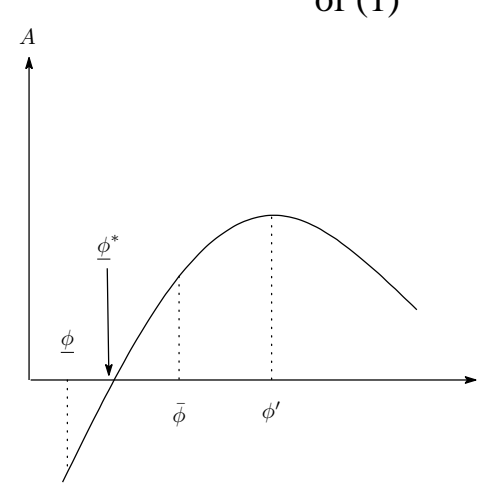

Figure 8: $2 k\left(1+\lambda_{a}\right) /\left(1+\lambda_{a}+\lambda_{q}\right)>\gamma:$ sketch of (2)

\section{Proof of Proposition 2.6}

We begin to prove (1). Hence, we show that $a^{* *}-a^{*}$ is positive under the condition. 
$a^{* *}-a^{*}$ is given as,

$$
\frac{t\left(k\left(1+\lambda_{a}\right)-\gamma\left(1+\lambda_{a}+\lambda_{q}\right)\right)}{k \gamma\left(1+\lambda_{a}\right)}-\frac{4 t^{2}\left(k\left(1+\lambda_{a}\right)-\gamma\left(1+\lambda_{a}+\lambda_{q}\right)\right) \phi}{\gamma^{2}\left(1+\lambda_{q}\right)^{2}} .
$$

This equation is a linear function of $\phi$ and a downward slope under the condition. For any $\phi$, this equation is positive. We can similarly prove (2).

\section{Proof of Proposition 2.7}

We show that $C S^{* *}-C S_{r}^{*}$ is a positive. $C S^{* *}-C S_{r}^{*}$ is given as

$$
\frac{\left(\gamma\left(1+\lambda_{q}\right)^{2}-4 k t\left(1+\lambda_{a}\right) \phi\right)^{2}}{4 \gamma^{2}\left(1+\lambda_{q}\right)^{2} \phi}>0
$$

\section{Proof of Proposition 2.8}

We show that $\pi^{* *}-\pi_{r}^{*}$ is a negative. The difference between $\pi^{* *}$ and $\pi_{r}^{*}$ is

$$
-\frac{\left(\gamma\left(1+\lambda_{q}\right)^{2}-4 k t\left(1+\lambda_{a}\right) \phi\right)^{2}}{8 \gamma^{2}\left(1+\lambda_{q}\right)^{2} \phi}<0
$$

\section{Proof of Proposition 2.9}

The difference between $C S^{*}$ and $C S^{* *}$ is given as follows.

$$
\begin{aligned}
& C S^{*}-C S^{* *}=\frac{1}{12 k \gamma^{2}\left(1+\lambda_{a}\right)\left(1+\lambda_{q}\right)^{2} \phi} \\
& \times\left\{-\gamma^{2}\left(1+\lambda_{q}\right)^{4}\left(3 k-2 \gamma+3 k \lambda_{a}-2 \gamma \lambda_{a}-2 \gamma \lambda_{q}\right)\right. \\
& +24 k t \gamma\left(1+\lambda_{a}\right)\left(1+\lambda_{q}\right)^{2}\left(k-\gamma+k \lambda_{a}-\gamma \lambda_{a}-\gamma \lambda_{q}\right) \phi \\
& \left.-48 k^{2} t^{2}\left(1+\lambda_{a}\right)^{2}\left(k-\gamma+k \lambda_{a}-\gamma \lambda_{a}-\gamma \lambda_{q}\right) \phi^{2}\right\} .
\end{aligned}
$$

The sign of this equation is equivalent to that of the numerator. Hence, we focus on the numerator. Note that the numerator is a quadratic function with respect to $\phi$. Let $F(\phi)$ be the numerator. The following lemma represents the property of $F(\phi)$.

Lemma A.2. $F(\phi)$ is monotonically increasing or decreasing in the region of $\phi<\left(\gamma\left(1+\lambda_{q}\right)^{2}\right) /(4 k t(1+$ $\left.\left.\lambda_{a}\right)\right)$ 
Proof. Solving $F^{\prime}(\phi)=0$ with respect to $\phi$, the extreme point $\phi^{*}$ of $F(\phi)$ is given as:

$$
\phi^{*}=\frac{\gamma\left(1+\lambda_{q}\right)^{2}}{4 k t\left(1+\lambda_{a}\right)}
$$

$\phi^{*}$

We begin to prove (1) of the proposition. By the lemma, we have the fact that the numerator is a monotone function in the region of $\phi \in\left(0,\left(\gamma\left(1+\lambda_{q}\right)^{2}\right) /\left(4 k t\left(1+\lambda_{a}\right)\right)\right.$. Note that $\phi$ is in the region of $\left[\underline{\phi}^{\text {non }}, \bar{\phi}^{\text {non }}\right]$ in this section, and $\bar{\phi}^{\text {non }}<\phi^{*}$. Therefore, $F(\phi)$ is a monotone function for any $\phi \operatorname{in}\left[\underline{\phi}^{\text {non }}, \bar{\phi}^{\text {non }}\right]$.

Substituting $\underline{\phi}^{\text {non }}$ into $F(\phi)$, we get

$$
F\left(\underline{\phi}^{\text {non }}\right)=\frac{1}{27} \gamma^{2}\left(1+\lambda_{q}\right)^{4}\left(22 \gamma\left(1+\lambda_{a}+\lambda_{q}\right)-49 k\left(1+\lambda_{a}\right)\right) \text {. }
$$

If $k<\left(22 \gamma\left(1+\lambda_{a}+\lambda_{q}\right)\right) /\left(49\left(1+\lambda_{a}\right)\right)$, then the equation is positive. Substituting $\bar{\phi}^{\text {non }}$ into $F(\phi)$, we have

$$
F\left(\bar{\phi}^{\mathrm{non}}\right)=\frac{1}{3} \gamma^{2}\left(1+\lambda_{q}\right)^{4}\left(2 \gamma\left(1+\lambda_{a}+\lambda_{q}\right)-5 k\left(1+\lambda_{a}\right)\right) .
$$

If $k<\left(2 \gamma\left(1+\lambda_{a}+\lambda_{q}\right)\right) /\left(5\left(1+\lambda_{a}\right)\right)$, then the equation is positive.

$F\left(\phi^{\text {non }}\right)$ and $F\left(\bar{\phi}^{\text {non }}\right)$ are positive if and only if $k<\left(2 \gamma\left(1+\lambda_{a}+\lambda_{q}\right)\right) /\left(5\left(1+\lambda_{a}\right)\right)$. Because $F(\phi)$ is a monotone function, $C S^{*}>C S^{* *}$ if $k<\left(2 \gamma\left(1+\lambda_{a}+\lambda_{q}\right)\right) /\left(5\left(1+\lambda_{a}\right)\right)$.

We prove, in turn, (2) of the proposition. Next, in $\left(2 \gamma\left(1+\lambda_{a}+\lambda_{q}\right)\right) /\left(5\left(1+\lambda_{a}\right)\right)<k<\left(22 \gamma\left(1+\lambda_{a}+\right.\right.$ $\left.\left.\lambda_{q}\right)\right) /\left(49\left(1+\lambda_{a}\right)\right), F(\phi)$ is positive in the region of $\left(\underline{\phi}^{\text {non }}, \phi^{\star \star}\right)$ but is negative in the region of $\left(\phi^{\star \star}, \bar{\phi}^{\text {non }}\right)$, where $\phi^{\star \star}$ is $\phi$ such that $C S^{*}=C S^{* *}$. Thus, if $k$ is in $\left(\left(2 \gamma\left(1+\lambda_{a}+\lambda_{q}\right)\right) /\left(5\left(1+\lambda_{a}\right)\right),\left(22 \gamma\left(1+\lambda_{a}+\right.\right.\right.$ $\left.\left.\left.\lambda_{q}\right)\right) /\left(49\left(1+\lambda_{a}\right)\right)\right)$, then $C S^{*}>C S^{* *}$ in the region of $\left(\underline{\phi}^{\text {non }}, \phi^{\star}\right)$ and $C S^{*}<C S^{* *}$ in the region of $\left(\phi^{\star}, \bar{\phi}^{\text {non }}\right)$.

We lastly prove (3) of the proposition. In the case of $k>\left(22 \gamma\left(1+\lambda_{a}+\lambda_{q}\right)\right) /\left(49\left(1+\lambda_{a}\right)\right), F(\phi)$ is negative in the assumed region of $\phi$. Therefore, $C S^{*}<C S^{* *}$.

\section{B Proofs in section 3}

\section{Proof of Proposition 3.1}

We begin to prove the case of (1). 
$\triangle C S$ is given as

$$
\begin{aligned}
\triangle C S & =\frac{1}{\left(4 t\left(\gamma-4 \gamma \lambda^{2}+16 k t \lambda \phi\right)^{2}\left(-\gamma \lambda-10 \gamma \lambda^{2}-24 \gamma \lambda^{3}-16 \gamma \lambda^{4}+3 k t \phi+28 k t \lambda \phi+80 k t \lambda^{2} \phi+64 k t \lambda^{3} \phi\right)^{2}\right)} \\
& \times\left((t+2 v \lambda)^{2}\right. \\
& \times \underbrace{\left(\gamma^{2}(1+2 \lambda)^{2}\left(1+8 \lambda+8 \lambda^{2}\right)-4 k t \gamma(1+2 \lambda)\left(3+17 \lambda+16 \lambda^{2}\right) \phi+8 k^{2} t^{2}(1+4 \lambda)(3+4 \lambda) \phi^{2}\right)}_{=\bar{A}(\phi)} \\
& \times \underbrace{\left(-\gamma^{2} \lambda(1+2 \lambda)^{2}\left(1+8 \lambda+8 \lambda^{2}\right)\right.}_{=b} \\
& -\underbrace{\left.\left.\left.2 k t \gamma(1+2 \lambda)\left(-3-22 \lambda-38 \lambda^{2}+32 \lambda^{3}+64 \lambda^{4}\right) \phi+8 k^{2} t^{2} \lambda(1+4 \lambda)(3+4 \lambda)(3+8 \lambda) \phi^{2}\right)\right)\right)}_{=b^{\prime}} .
\end{aligned}
$$

Let $b-b^{\prime}$ be $B(\phi)$. Note that both $\bar{A}(\phi)$ and $B(\phi)$ are quadratic functions with respect to $\phi$. We can characterize $\triangle C S$ by the conditions of the signs of both $\bar{A}(\phi)$ and $B(\phi)$, since the denominator and $(t+2 v \lambda)^{2}$ are positive. Hence, we evaluate the conditions of the signs of both $\bar{A}(\phi)$ and $B(\phi)$.

Lemma B.1. The function $\bar{A}(\phi)$ is monotonically decreasing in $\phi \in[\underline{\phi}, \bar{\phi}]$.

Proof. When we differentiate $\bar{A}(\phi)$ with respect to $\phi$, we get

$$
-4 k t \gamma(1+2 \lambda)\left(3+17 \lambda+16 \lambda^{2}\right)+16 k^{2} t^{2}(1+4 \lambda)(3+4 \lambda) \phi
$$

Let the differential coefficient of the first order be $\bar{A}^{\prime}(\phi)$. Moreover, the differential coefficient of the second order of $\bar{A}(\phi)$ is

$$
16 k^{2} t^{2}(1+4 \lambda)(3+4 \lambda)
$$

Thus, the second-order condition is a positive. Let the differential coefficient of the second order be $\bar{A}^{\prime \prime}(\phi)$.

Solving $\bar{A}^{\prime}(\phi)=0$ with respect to $\phi$,

$$
\phi^{\prime}=\frac{\gamma(1+2 \lambda)\left(3+17 \lambda+16 \lambda^{2}\right)}{4 k t\left(3+16 \lambda+16 \lambda^{2}\right)} .
$$

Note that $\phi^{\prime} \in \operatorname{argmin} \bar{A}(\phi)$ since $\bar{A}^{\prime \prime}(\phi)>0$. Calculating $\bar{\phi}-\phi^{\prime}$, we get

$$
-\frac{3 \gamma \lambda(1+2 \lambda)}{4 k t(1+4 \lambda)(3+4 \lambda)}
$$

Thus, $\bar{\phi}<\phi^{\prime}$. Moreover, since $\bar{A}(\phi)$ is convex, $\bar{A}(\phi)$ is monotonically decreasing in the region of $\phi$. 
Lemma B.2. The function $B(\phi)$ is monotonically increasing in $\phi \in[\phi, \bar{\phi}]$.

Proof. When we differentiate $B(\phi)$ with respect to $\phi$, we get

$$
-2 k t \gamma(1+2 \lambda)\left(-3-22 \lambda-38 \lambda^{2}+32 \lambda^{3}+64 \lambda^{4}\right)+16 k^{2} t^{2} \lambda(1+4 \lambda)(3+4 \lambda)(3+8 \lambda) \phi .
$$

Let the differential coefficient of first order be $B^{\prime}(\phi)$. Moreover, the differential coefficient of second order of $B(\phi)$ is

$$
16 k^{2} t^{2} \lambda(1+4 \lambda)(3+4 \lambda)(3+8 \lambda)
$$

Thus, second order condition is positive. Let the differential coefficient of second order be $B^{\prime \prime}(\phi)$. Solving $B^{\prime}(\phi)=0$ with respect to $\phi$,

$$
\phi^{\prime \prime}=\frac{\gamma(1+2 \lambda)\left(-3-22 \lambda-38 \lambda^{2}+32 \lambda^{3}+64 \lambda^{4}\right)}{8 k t \lambda(3+8 \lambda)\left(3+16 \lambda+16 \lambda^{2}\right)} .
$$

Note that $\phi^{\prime} \in \operatorname{argmin} B(\phi)$ since $B^{\prime \prime}(\phi)>0$. Calculating $\underline{\phi}-\phi^{\prime \prime}$, we get

$$
\frac{\gamma(1+2 \lambda)\left(9+126 \lambda+738 \lambda^{2}+2336 \lambda^{3}+4000 \lambda^{4}+3328 \lambda^{5}+1024 \lambda^{6}\right)}{8 k t \lambda(1+4 \lambda)^{2}(3+4 \lambda)^{2}(3+8 \lambda)} .
$$

Thus, $\underline{\phi}>\phi^{\prime \prime}$. Moreover, since $B(\phi)$ is convex, $B(\phi)$ is monotonically increasing in the region of $\phi$.

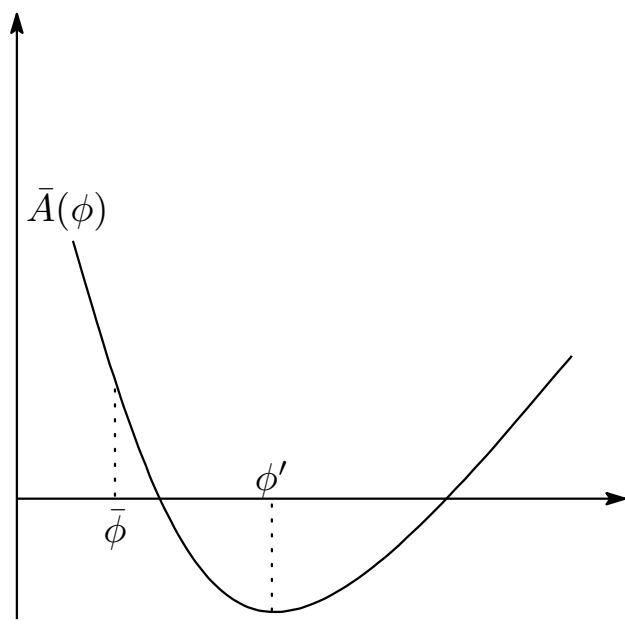

Figure 9: Sketch of lemma 3

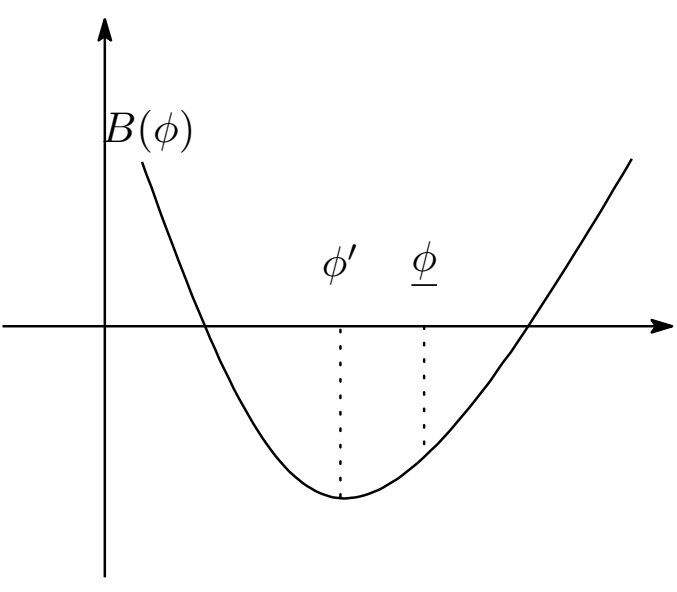

Figure 10: Sketch of lemma 4 
Substituting $\phi$ and $\bar{\phi}$ for $\bar{A}(\phi)$, we get

$$
\begin{aligned}
& A(\underline{\phi})=\frac{\gamma^{2}(1+2 \lambda)^{3}\left(1+8 \lambda+8 \lambda^{2}\right)\left(11+116 \lambda+472 \lambda^{2}+1024 \lambda^{3}+1152 \lambda^{4}+512 \lambda^{5}\right)}{(1+4 \lambda)^{3}(3+4 \lambda)^{3}}, \\
& A(\bar{\phi})=-\frac{\gamma^{2}(1+2 \lambda)^{2}\left(3+22 \lambda+49 \lambda^{2}+32 \lambda^{3}\right)}{2(1+4 \lambda)(3+4 \lambda)}
\end{aligned}
$$

Since a discriminant of $A(\phi)$ is a positive, $\operatorname{sign}(\bar{A}(\underline{\phi})) \neq \operatorname{sign}(\bar{A}(\bar{\phi}))$, and convex, there exists $\phi^{*} \in[\underline{\phi}, \bar{\phi}]$ such that $\bar{A}(\phi)=0 .{ }^{6}$ Let $\phi^{*}$ be $\phi^{*}$.

Also, substituting $\underline{\phi}$ and $\bar{\phi}$ for $B(\phi)$, we get

$$
\begin{aligned}
& B(\underline{\phi})=\frac{\gamma^{2}(1+2 \lambda)^{3}\left(1+8 \lambda+8 \lambda^{2}\right)\left(9+147 \lambda+932 \lambda^{2}+2968 \lambda^{3}+5120 \lambda^{4}+4480 \lambda^{5}+1536 \lambda^{6}\right)}{(1+4 \lambda)^{3}(3+4 \lambda)^{3}}, \\
& B(\bar{\phi})=\frac{\gamma^{2}(1+2 \lambda)^{2}\left(3+9 \lambda+8 \lambda^{2}\right)\left(3+22 \lambda+40 \lambda^{2}+32 \lambda^{3}\right)}{2(3+4 \lambda)} .
\end{aligned}
$$

By both $B(\underline{\phi})$ and $B(\bar{\phi})$ are positives, and the above lemma $B(\phi)$ is positive in the region of $\phi$.

In the region of $\left[\underline{\phi}, \underline{\phi}^{*}\right]$, both $\bar{A}(\phi)$ and $B(\phi)$ are positives. Thus, $\triangle C S$ is positive.

We prove the case of (2). By the logic of the proof for (1), in the region of $\left[\underline{\phi}^{*}(\bar{A}), \bar{\phi}\right], \bar{A}$ is a negative and $B$ is a positive. Therefore, $\triangle C S$ is a negative.

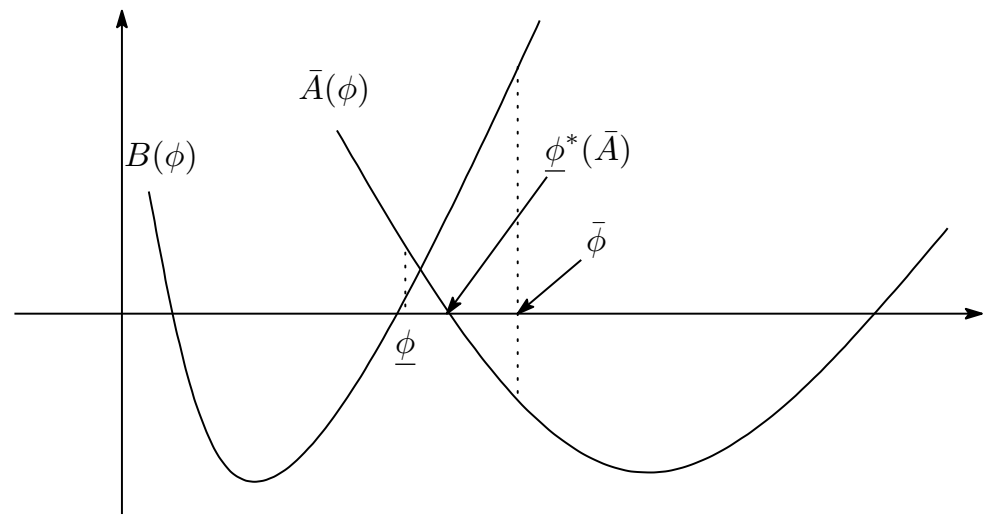

Figure 11: Sketch of Proposition 3.1

\footnotetext{
${ }^{6} \operatorname{sign}(\cdot)$ maps from the value to the sign. For example, $\operatorname{sign}(5)=+$.
} 


\section{Comparison of programs quality of non-self-regulation with self-regulation}

Proposition C.1. In three types model in section $2, q_{i}^{*}>q_{r}^{*}$ for all $\phi \in\left(\underline{\phi}^{\text {non }}, \bar{\phi}^{\text {non }}\right)$.

Proof. We evaluate $\phi$ such that $q_{i}^{*}=q_{r}^{*}$. The $\phi$ is given as

$$
\tilde{\phi}=\frac{\gamma\left(1+\lambda_{q}\right)^{2}}{6 k t\left(1+\lambda_{a}\right)}
$$

If $\tilde{\phi}>\phi$, then the difference is positive. Note that the difference between $q_{i}^{*}$ and $q_{r}^{*}$ is decreasing function of $\phi$. Since $\tilde{\phi}$ is larger than $\bar{\phi}^{\text {non }}$, for any $\phi$ in the region the difference is positive.

Proposition C.2. In two types of models in section 3

(i) $q_{i}^{*}>q_{r}^{*}$ for all $\phi \in(\underline{\phi}, \tilde{\phi})$.

(ii) $q_{i}^{*}<q_{r}^{*}$ for all $\phi \in(\tilde{\phi}, \bar{\phi})$.

Proof. We evaluate $\phi$ such that $q_{i}^{*}=q_{r}^{*}$. The $\phi$ is given as

$$
\tilde{\phi}=\frac{\gamma(1+2 \lambda)(1+8 \lambda(1+\lambda))}{2 k t(3+4 \lambda(5+4 \lambda))} .
$$

If $\tilde{\phi}>\phi$, then the difference is positive. Note that the difference between $q_{i}^{*}$ and $q_{r}^{*}$ is a decreasing function of $\phi$. Since $\phi$ is smaller than $\tilde{\phi}$ and $\bar{\phi}$ is larger than $\tilde{\phi}$, on $\tilde{\phi}$ the difference changes to negative from positive.

Note C.3. In two types of models in section 3, $\tilde{\phi}>\underline{\phi}^{*}$. Thus, in the region of $\phi$ in Proposition 3.1 - (i), $q_{r}^{*}<q_{i}^{*}$.

Proof. We can proof this statement by using lemma B.1. Since, as with $\phi^{*}, A(\phi)$ changes to negative from positive; if the sign of $A(\tilde{\phi})$ is negative, then $\phi^{*}<\tilde{\phi}$. $A(\tilde{\phi})$ is given by

$$
-\frac{(\gamma+2 \gamma \lambda)^{2}(1+8 \lambda(1+\lambda))(3+2 \lambda(11+4 \lambda(5+4 \lambda)))}{(3+4 \lambda(5+4 \lambda))^{2}} .
$$

Since this value is negative, we get $\phi^{*}<\tilde{\phi}$. 